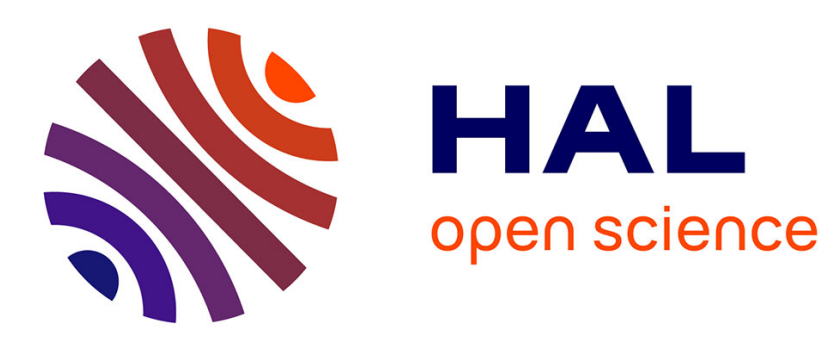

\title{
Specifications and conceptual architecture of a thermal infrared simulator of landscapes
}

Thierry Poglio, Eric Savaria, Lucien Wald

\section{To cite this version:}

Thierry Poglio, Eric Savaria, Lucien Wald. Specifications and conceptual architecture of a thermal infrared simulator of landscapes. Colloquium SPIE (EUROPTO) "Sensors, Systems, and Next Generation Satellites VII" (CD-ROM), Sep 2001, Toulouse, France. pp.488-497. hal-00395025

\section{HAL Id: hal-00395025 \\ https://hal.science/hal-00395025}

Submitted on 14 Jun 2009

HAL is a multi-disciplinary open access archive for the deposit and dissemination of scientific research documents, whether they are published or not. The documents may come from teaching and research institutions in France or abroad, or from public or private research centers.
L'archive ouverte pluridisciplinaire HAL, est destinée au dépôt et à la diffusion de documents scientifiques de niveau recherche, publiés ou non, émanant des établissements d'enseignement et de recherche français ou étrangers, des laboratoires publics ou privés. 


\title{
Specifications and conceptual architecture of a thermal infrared simulator of landscapes
}

\author{
Thierry Poglio ${ }^{* a, b}$, Eric Savaria ${ }^{* a}$, Lucien Wald ${ }^{* * b}$ \\ ${ }^{\mathrm{a}}$ Alcatel Space, System Architecture Division; ${ }^{\mathrm{b}}$ Groupe "Télédétection \& Modélisation”, Ecole des \\ Mines de Paris
}

\begin{abstract}
This paper deals with the simulation of very high spatial resolution images in the thermal infrared range, from 3 to 14 $\mu \mathrm{m}$. It focuses on the conceptual architecture of a simulator of 3-D landscapes; its specifications are described and discussed. A new methodology is proposed for the simulation to best reproduce the properties of the infrared imagery. Particularly, this methodology enables a very accurate simulation of the signal coming from each object constituting the landscape. The interactions between the radiations and objects and between objects themselves are considered. Their changes in time, and the recent past of the temperature and the humidity for each object, are taken into account. To reproduce these physical phenomena, the computation is performed on elements, which are defined as homogeneous entities with respect to the physical processes. This concept of element leads to a new methodology in design and realization of simulators. It permits to reproduce efficiently the behavior of the landscape in this spectral range at very high spatial resolution.
\end{abstract}

Keywords: Infrared, simulator design, 3-D scene, very high spatial resolution, object interactions.

\section{INTRODUCTION}

There is a large demand of very high spatial resolution imagery in the infrared range from 3 to $14 \mu \mathrm{m}$. Various fields, like meteorology, farming or military information are concerned. Yet, such imagery with a spatial resolution of a meter or so is not available: new spaceborne systems have to be designed. Critical points are the assessment of the capacity of such systems and users training to such images. The simulation is a crucial tool in this respect and helps in reproducing the characteristics of the acquisition system. It produces an image such as it would have been observed by the acquisition system. One of the major points in the simulation is the accurate knowledge of the input parameters, and particularly the accurate knowledge of the scene the system has to observe. A simulator of landscapes enables this necessary knowledge.

Changing meteorological conditions, different places, different landscapes, different times and different spectral bands should be simulated. A landscape synthesis method was selected in order to meet better these requirements. In thermal infrared, the flux coming from an object is partly emitted by the object because of its own temperature, and partly due to the reflection of incident rays on the surface of this object. Depending on the surface material and the spectral band, emission or reflection process dominates the signal. For each object in the scene, the landscape simulator predicts the heat exchanges between objects, the temporal evolution of heat balance, the surface temperature, the spectral emission and the spectral reflection of all incident fluxes. Such simulator takes into account 3-D landscape description, environmental conditions, thermal and optical characteristics of the objects, and the spectral band of the sensor.

Jaloustre-Audouin (1998) and Jaloustre-Audouin et al. (1997) have developed a simulator of any type of landscape in 2$\mathrm{D}$ in the infrared band. Image simulators taking into account a 3-D representation of the landscape as input exist, but they are for visible range or dedicated to specific applications. For example, Thirion (1991) has developed a simulator

\footnotetext{
* thierry.poglio@space.alcatel.fr; phone +33 (0)4 929264 24; fax +33 (0)4 929276 60; Alcatel Space Industries, 156 Bd. Du Midi, 06156 Cannes-la-Bocca, France;

** lucien.wald@ensmp.fr; phone +33 (0)4 939574 49; fax +33 (0)4 939575 35; Ecole des Mines de Paris, B.P. 207, 06904 Sophia Antipolis Cedex, France.
} 
of high spatial resolution image in the visible range. Johnson et al. (1998) have developed a simulator in the infrared range to simulate the behavior of vehicles. Guillevic (1999) was interested in radiative budget modeling for vegetation canopy studies. Barillot (2001) has developed MISTRAL, which can simulate an image taking into account a 3-D description of the landscape. This simulator of landscape deals with scenes of small size. A simulator of landscape adapted to large scenes for remote sensing applications does not exist and has to be developed. It should take into account a 3-D description of the scene with high spatial resolution description, and consider scenes with a size of a few kilometers.

Physical processes playing a part in the signal coming from the scene are described in the following section. Next, the synthesis method applied to the simulation of landscape with a 3-D representation in the infrared range is discussed. The new methodology proposed for the simulation of very high-resolution 3-D scene, and the consequences on the landscape modeling are explained in section 4. Section 5 details the architecture of the simulator using this modeling; each subpart of the simulator is presented, and its operating is explained.

\section{PHYSICAL PROCESSES}

In thermal infrared, the flux coming from an object in a given spectral range is both due to its own temperature and to spectral reflection of incident fluxes in this spectral range. Depending on the spectral band and the surface material, the emission or reflection process dominates the signal. So, both processes have to be computed carefully. Nevertheless, considering only order of importance, reflection process dominates the signal in band II during the day whereas emission process dominates in band III whatever the considered moment in the day is.

\subsection{The emitted flux}

The emitted flux or irradiance (in W. $\mathrm{m}^{-2}$ ) received by a sensor with a spectral response $g(\lambda)$ in a given spectral range, from $\lambda_{1}$ to $\lambda_{2}$ is expressed as

$$
L_{\lambda_{1} \lambda_{2}}^{e}\left(\theta_{v}, \varphi_{v}\right)=\pi \int_{\lambda_{1}}^{\lambda_{2}} g(\lambda) \varepsilon_{s}\left(\lambda, \theta_{v}, \varphi_{v}\right) L^{b b}\left(\lambda, T_{s}\right) d \lambda
$$

with

$$
L^{b b}\left(\lambda, T_{s}\right)=\frac{2 \cdot h c^{2}}{\lambda^{5} \cdot\left[\exp \left(\frac{h c}{\lambda k T_{s}}\right)-1\right]}
$$

where $L^{b b}$ is the isotropic flux emitted by a blackbody. $T_{s}$ is the surface temperature of the considered material, and $\left(\theta_{v}, \varphi_{v}\right)$ the angle of the viewing direction. $h, c$ and $k$ are respectively the Planck constant, the light velocity and the Boltzmann constant. The quantity $\varepsilon_{s}$ is the spectral emissivity of the object. The knowledge of the spectral emissivity and of the surface temperature of the material enables the knowledge of emitted flux.

\subsection{The reflected flux}

All incident fluxes coming from other objects in the scene have to be considered as potential sources, especially in the infrared range where each object emits a flux due to is own temperature. An object $i$ reflects a part of the received radiations $H_{i}$ of various origin:

- solar radiation,

- atmospheric emission,

- reflected and emitted radiations from the surrounding objects.

The flux $L_{i}^{r}$ reflected by the object $i$ is given by

$$
L_{\lambda_{1} \lambda_{2}}^{r}\left(\theta_{v}, \varphi_{v}\right)=\pi \int_{\lambda_{1}}^{\lambda_{2}} g(\lambda) f_{r}\left(\lambda, \theta, \varphi, \theta_{v}, \varphi_{v}\right) H_{i}(\lambda, \theta, \varphi) d \lambda
$$


where $f_{r}\left(\lambda, \theta, \varphi, \theta_{v}, \varphi_{v}\right)$ is the bidirectional reflectance distribution function (BRDF, symbol $f_{r}$ ). It is defined as the differential element of reflected radiance in a specified direction per unit differential element of radiant incidence, also in a specified direction (Nicodemus et al., 1977), and is expressed in $\operatorname{sr}^{-1} .\left(\theta_{v}, \varphi_{v}\right)$ are angles of the viewing direction, and $(\theta, \varphi)$ those of the incident radiation. The contribution of the surrounding, expressed by $H_{i}$, can be written as a sum of incident fluxes, each flux coming from a neighbor of the object $i$. Neighbors include the atmosphere and the Sun, which can be considered as neighbors for all objects. Using this formulation, the global contribution of the surrounding is expressed as

$$
{ }^{L_{i}^{r} \lambda_{1} \lambda_{2}}\left(\theta_{v}, \varphi_{v}\right)=\sum_{j=1}^{N} \frac{1}{A_{i}} \int_{A_{i}} \int_{A_{j}} \int_{\lambda_{1}}^{\lambda_{2}} g(\lambda) \xi\left(\lambda, \theta_{i j}, \varphi_{i j}, r_{i j}\right) f_{r}\left(\lambda, \theta_{i j}, \varphi_{i j}, \theta_{v}, \varphi_{v}\right) B_{j}\left(\lambda, \theta_{i j}, \varphi_{i j}\right) \cos \theta_{i j} \frac{\cos \theta_{j}^{\prime}}{r_{i j}^{2}} V_{i j} d A_{i} d A_{j} d \lambda
$$

In this equation, $\xi$ is the function expressing the atmospheric transmission, depending on the distance between the considered objects and on the objects viewing angles. The transmission is also depending on the spectral band. The $B_{j}$ function represents the global flux coming from the object $j$, seen with the direction $\left(\theta_{i j}, \varphi_{i j}\right)$ from the object $i$. $A_{i}$ and $A_{j}$ are the surfaces of the objects $i$ and $j$.

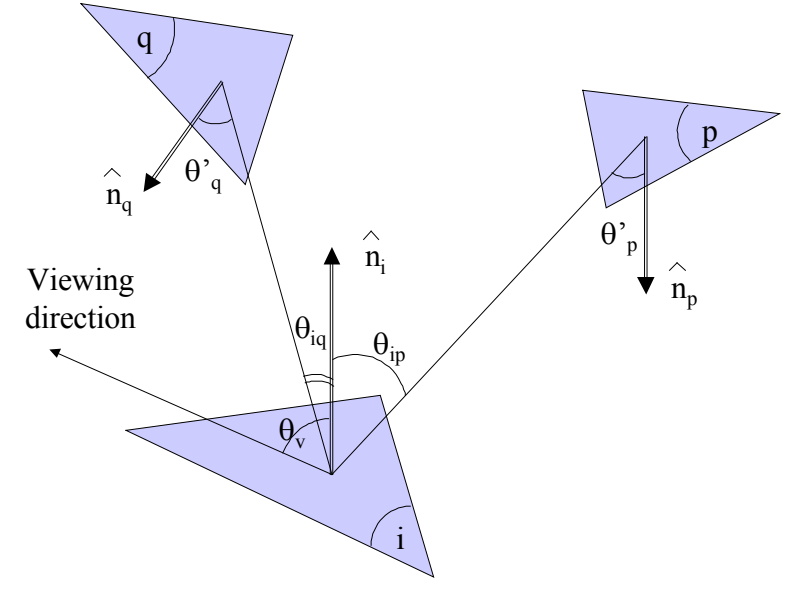

Figure 1: illustration of the different angles playing a part and notations used for the multiple reflections. The $n_{p}$ vector is the orthogonal vector for the object $p$; for the sake of simplicity, only the zenithal angles $\theta$ are drawn.

The different angles used in the equation 4 are illustrated by the figure 1 . The $V_{i j}$ term expresses the visibility between two objects: it is equal to 1 if objects are totally visible, 0 if they are without visibility, and between 0 and 1 if they are partially visible. $\mathrm{N}$ denotes the number of neighbors for the considered object $i$.

In the equation 4 , the $B_{j}$ fluxes are unknowns as well as $L_{i}^{r}$. The computation of the fluxes $L_{i}^{r}$ for all objects requires solving a non-linear system of equations, the number of equations being equal to the number of unknowns $L_{i}^{r}$. Several methods exist to solve numerically such a problem, each of them making various assumptions to facilitate the resolution of the system (Foley, 1996). For example, the radiosity method assumes ideal diffuse reflectors and ideal diffuse emitters (Watt, 2000; Sillion and Puech, 1994). In this method, the incoming radiances are the only directional data, and spectral data cannot be taken into account.

Whatever the methodology used and the assumptions made to solve equation 4, this equation demonstrates that the computation of the reflected flux received by an object requests the knowledge of the distribution and the orientation of the objects in the surrounding and their interactions.

\section{THE SYNTHESIS}

In the infrared range, the spectral flux coming from an object depends upon the meteorological conditions existing in the scene: spectral range, surface temperature, air temperature, humidity of the object, humidity of the atmosphere... 
and the optical properties of the object itself. A process for synthesis of scene was selected. It is more appropriate to the predictions of the spectral flux coming for an object in any case, compared to approaches dealing with images acquired over real scenes in specific conditions. Changing meteorological conditions, different places, different landscapes, different times and different spectral bands should be simulated. The figure (1) illustrates the mechanism of the image synthesis in the infrared range.

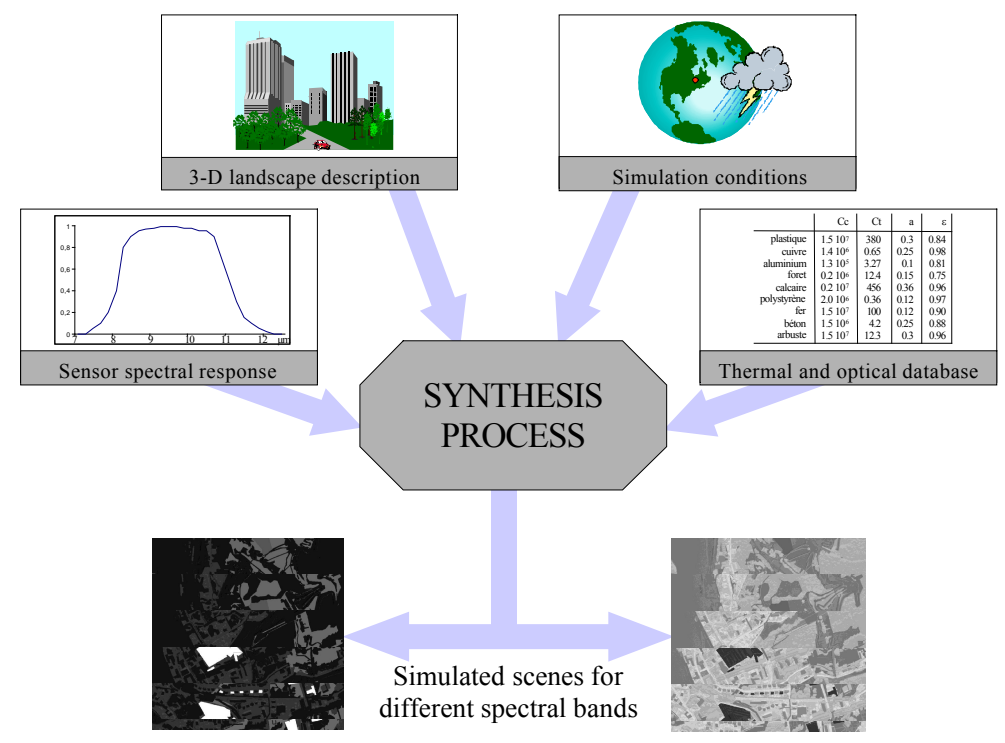

Figure 2: illustration of the synthesis process in the infrared range; the user inputs and the simulator outputs.

To perform a simulation, the user must give inputs to the simulator. These inputs can be divided into four parts:

- the 3-D landscape geometrical description. The landscape is expressed as a set of located and oriented objects, having their own surrounding. Each object is built with several facets, each of them made with the same material,

- the set of the conditions of the simulation for the present time and past hours: day, time, place, precipitation, sky cloudiness...,

- $\quad$ a database of thermal and optical characteristics of all primary materials existing in the landscape (albedo, spectral reflectance (ASTER, 2000), specific heat, thermal conductivity, roughness, leaf area index for vegetation,...),

- $\quad$ the spectral response of the sensor; this is a necessary input to compute emitted and reflected flux for the objects which constitute the landscape. The knowledge of the point-spread function (or the modulation transfer function) is required to simulate accurately the final image, as it would be seen by the sensor.

With all these requirements, the simulator will successively define its working environment, then compute global flux balance and surface temperature with an iterative method; at the moment of the simulation, it will predict the emitted flux and compute spectral reflectance of the landscape. Finally, depending on the viewing parameters given by the users, it will generate an image for any viewing conditions.

The final outputs of the simulator are images, generated for any viewing conditions, and for specified sensor point spread function. In addition, the simulator is a tool for the training of users. Several extra outputs exist corresponding to the different steps to better understand the synthesis process. These outputs will be discussed in the section 5 where the description of the simulator and the synthesis process are detailed.

\section{A NEW METHODOLOGY FOR THE SIMULATION}

An original design has been selected for the simulator to best reproduce the impacts of the most relevant physical phenomena on the synthesized scene.

The computation of the emitted flux at a given time $t$ requests the knowledge of the surface temperature at this instant (equation 1). Due to thermal inertia, the knowledge of this temperature at $t$ requests the temperature at the previous 
moment $(t-d t)$. Given an initial state, the temperature is computed using an iterative method; details are given in the next section. The interactions between physical parameters, and their variations in time are modeled. Methods exist (Johnson, 1995; Jaloustre-Audouin et al., 1997) to compute these interactions and change in time. These interactions differentiate the simulation in the infrared range from that in the visible range. The synthesis methods used for the simulation of landscapes for short wavelengths do not need to reproduce the recent past of the landscape as it is observed in real infrared images.

To reproduce this recent past a new concept was defined. This concept is based on intrinsic characteristics of the landscape during the synthesis. These characteristics permit to model the landscape by the means of homogeneous entities with respect to the physical phenomena occurring during the synthesis. To perform a simulation for short wavelengths, the landscape representation is usually a set of objects; each object is described by a set of facets on which the calculus of reflected flux is performed. This set of facets is a function of the 3-D description of the landscape; not only the object descriptions are important, but also their positioning in the landscape. In thermal infrared, during the synthesis process, surface temperature of each entity is evaluated at each iteration. This temperature depends not only on the temperature of the entity at the previous moment, but also on the boundary conditions existing on the entity. The prediction of a unique surface temperature for a given entity is only relevant if the same boundary conditions apply to each point of the entity. The entity should exhibit homogeneous properties with respect to the occurring physical phenomena at instant $t$. Among these phenomena, some are independent on the surface temperature (solar radiations, or deep temperature for example) whereas others depend unambiguously on this temperature (convective fluxes, radiative losses...). Surface temperature independent physical processes help to define homogeneous entities. Homogeneity means homogeneity at surface and in depth.

For example, the amount of solar radiation received at instant $t$ is equal across the surface of the entity. The same stands for the surface temperature and the in-depth temperature.

The most physically relevant phenomena are shadowing effects and wind disturbances around buildings (Poglio et al., 2001). Theoretically, wind flow, air temperature and surface temperature are related. In this study, for the sake of the simplicity wind flow is assumed to be independent on the temperature gradient existing between the ground and the atmosphere. This impedes the simulation of the advection of heat. These physical processes are intrinsic characteristics of the considered entity. Using these intrinsic characteristics on the one hand, and the facet characteristics (location, orientation, constitution) on the other hand, this homogeneous entity is be defined. This entity is called hereafter the element, on which all calculus are performed.

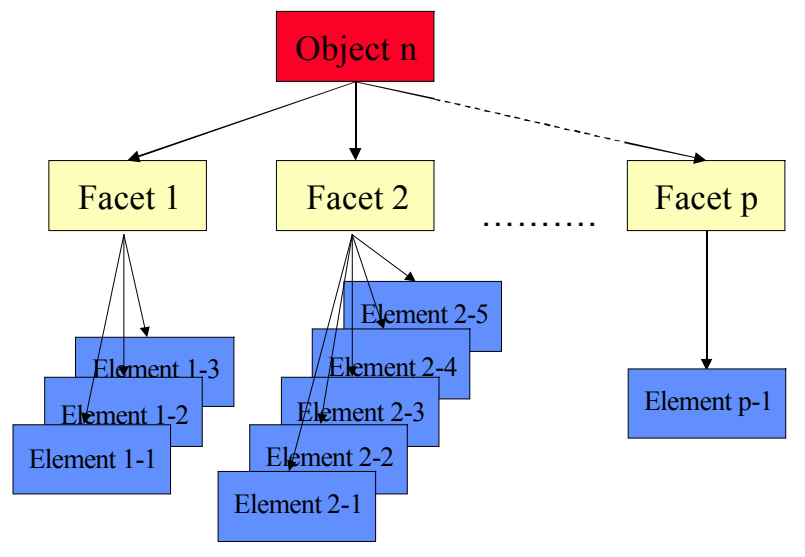

Figure 3: illustration of the landscape element representation; inheritance from object to facets, next from facets to elements. In each subdivision step, properties and characteristics of the entity are added to finally perform an element-based representation of the landscape. 
To better understand the element concept, one may consider a facet; this facet is partly shadowed at the instant $t$. These portions of the facet are a first set of elements, which will be subsequently subdivided by taking into account other phenomena and other instants.

The landscape can be expressed in elements. The figure 3 shows the different steps used to construct the elements from the objects. The element-based representation is performed by the simulator (S0) and is detailed in the following section.

\section{ARCHITECTURE OF THE SIMULATOR}

The simulator is divided into four primary simulators, each of them dealing with a particular sub-part of the simulation (figure 4). These four primary simulators, called (S0) to (S3), operate successively: the simulator (S0) defines and creates elements applying requirements explained above; (S1) computes the surface temperature; (S2) predicts the emitted and reflected fluxes for each element; (S3) generates an image with the appropriate point spread function in the specified spectral band.

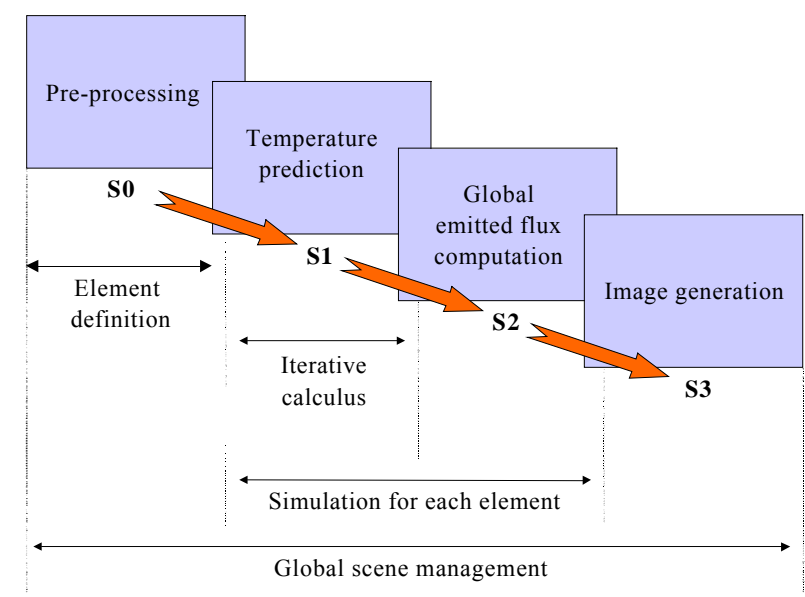

Figure 4: illustration of the global architecture of the simulator

The necessary inputs of the primary simulator $(\mathrm{S} 0)$ are:

- the ancillary data: the place and the time of the simulation, latitude, longitude, day and month in the year, and local time of the simulation. The meteorological parameters and their evolution during the synthesis have to be given: initial humidity in the ground, precipitation, air relative humidity and the minimal and maximal air temperatures.

- the scene description. The user must give an object-oriented scene description of the landscape. Location of buildings, houses, trees, roads, forests...must be given.

- material description. Each object in the landscape is built with different materials; using primary material database (plastic, concrete, cooper, sand, trees,...), the user can create as much material as he wants. For example, a facade may be created with a white paint without depth, $20 \mathrm{~cm}$ breeze-block, $5 \mathrm{~cm}$ polystyrene insulation, and $5 \mathrm{~cm}$ inner wall.

- $\quad$ surrounding definition rules. These rules are used for the determination of the neighbors (the conductive and the radiative neighbors) of an object.

Concerning the second and third point, the user can give to the simulator a facet-oriented representation of the landscape, each facet being labeled with the constituting material.

The originality of such a tool is the preprocessing simulator, (S0), defining homogeneous entities, with respect to geometry, constitution, and physical processes. The structure of the simulator (S0) is detailed in figure 5.

The simulator (S0) operates with the following scheme. Temporal evolution of solar irradiance is computed with the knowledge of the place, the meteorology, and the instant of the simulation. The shadow maps are made, and with the 
facet description of the scene, the element-based representation is obtained. With the facet scene description and the neighborhood determination rules, neighbors can be found with respect to respectively conduction process (conductive neighbors) and radiative process (radiative neighbors). The simulator does not look for convective neighbors: for wind flow computation through the scene, the global description of the scene is taken into account. The simulator is objectoriented: the neighbors of a given facet of a given object are found among other facets constituting the same object, or among facets constituting the objects belonging the surrounding of the considered object. The same procedure is applied between the facet-oriented description of the scene and the element-oriented one. After these procedures, each element has its own surrounding, described as a list of elements. The last procedure performed by the simulator is the form factor computation (Schröder, 1993). Finally, the five outputs of the preprocessing simulator are:

- $\quad$ an element-based representation, which will be used for all the following physical calculus (temperature, emitted and reflected fluxes),

- a radiative element surrounding description,

- a conductive element surrounding description,

- $\quad$ the knowledge of the wind velocity at the surface of each element,

- $\quad$ the form factor matrix, which will be used to compute radiative exchanges between elements.

All these outputs of the simulator (S0) are inputs for the simulator (S1), in addition to any of the first primary simulator (S0).

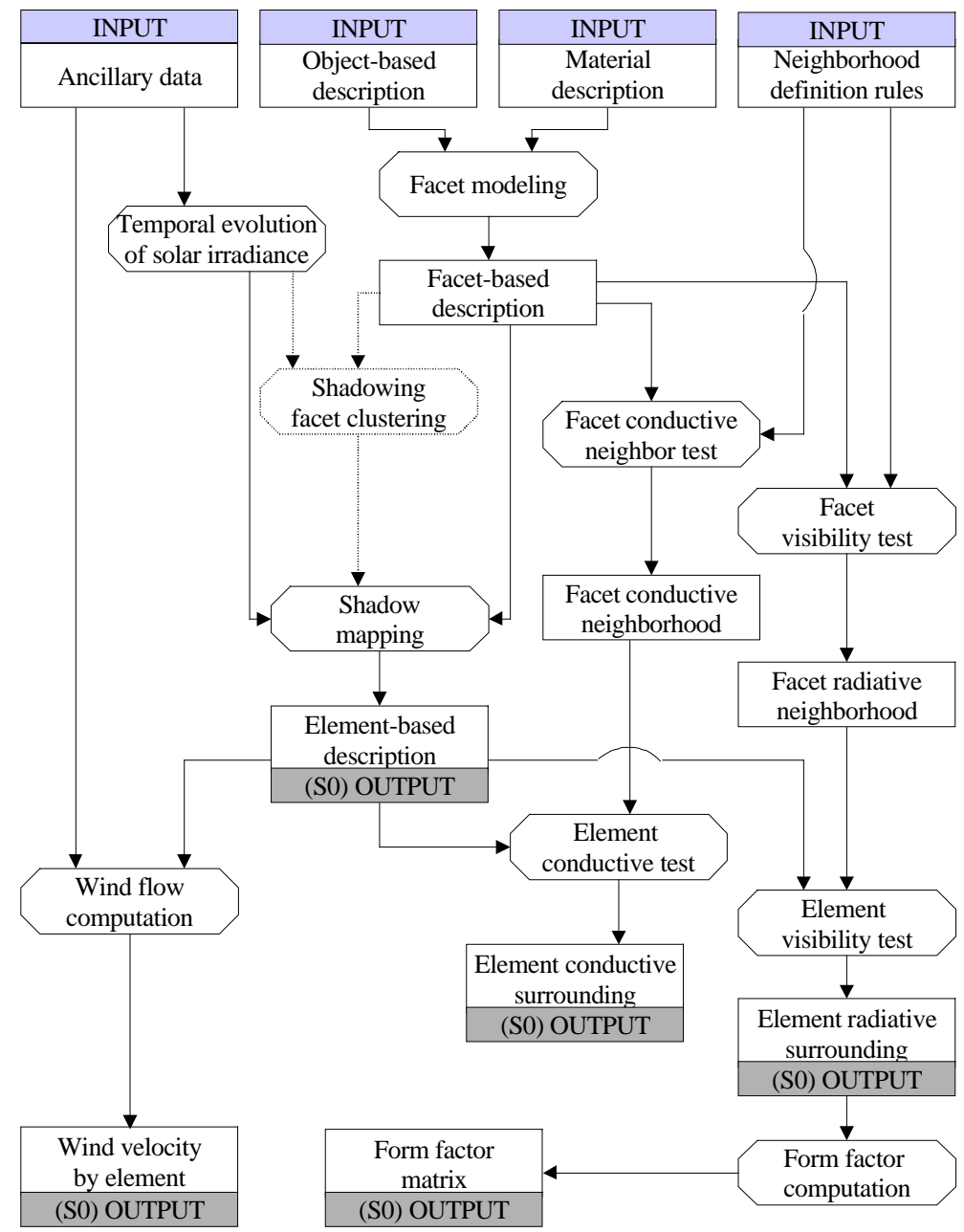

Figure 5: the detailed architecture of the (S0) primary simulator. In full lines, the required architecture to obtained elements; in dotted lines, the shadowing facet clustering, giving the opportunity to accelerate and to optimize the element creation. 
The second primary simulator (S1) predicts the surface temperature for each element defined by (S0). The simulator operates with iterations, from the initial time to the simulation time. The initial time is usually definite by the sunrise the previous day of the simulation time. At this initial time, the temperature of each element is supposed to be equal to the air temperature. Each element is preprocessed for the heat equation solving; each layer of the element made with a given material is divided into several strata to obey the convergence conditions. The heat equation can be solved by using e.g. the finite difference method or the Deardorff method (Deardorff, 1978). With help of models, the simulator predicts, for each time step:

- the heat exchanges,

- the relative humidity,

- $\quad$ the temperature on all the depth for each element.

Heat exchanges occur due to radiations, convection and conduction. Radiations can be computed with the modeling of the solar flux (Rigollier et al., 2000), the atmospheric emission (Olseth et al., 1994), and the surrounding of the elements. Convective fluxes require the knowledge of the temperature gradient between the ground and the air, the wind velocity (S0 output), the roughness of the material for sensible heat flux assessment (Louis, 1979). In addition, latent heat flux prediction requires the knowledge of the ground moisture (Noilhan and Planton, 1989). The heat conduction between elements is computed with the knowledge of intrinsic characteristics of the elements, like specific heat $c_{c}$ and thermal conductivity $c_{t}$, and with the knowledge of the positioning of the elements themselves ( $\mathrm{S} 0$ output). The heat equation balance, expressed as

$$
(\text { conductive flux })=(\text { radiative flux })-(\text { convective flux })
$$

permits to obtain one of the two boundary conditions required for heat equation and humidity equation solving. The second boundary condition is the knowledge of the deep temperature of each element, which is also an intrinsic parameter of the element representation. Heat equation (equation 6) and humidity equation can be solved for each time step, until the simulation time is reached.

$$
\frac{\partial T(\vec{r} ; t)}{\partial t}=\frac{c_{t}}{c_{c}} \cdot \Delta T(\vec{r} ; t)
$$

The output of the second primary simulator $(\mathrm{S} 1)$ is the surface temperature for each element constituting the landscape.

Then, the third primary simulator (S2) simulates spectral emitted and reflected flux. Emitted flux is predicted with the help of equation 1 and the (S1) output; ancillary data, like the viewing direction of the scene is required here, especially if the own emission of the considered element can not be considered as a lambertian emission. Reflected flux computation requires the knowledge of all incident spectral fluxes. Particularly, the Sun spectral radiation and the atmospheric spectral radiation have to be modeled; this can be performed by the code MODTRAN (Kneizys et al., 1988). As equation 4 is not easy to solve, a first assumption is done, considering that the spectral flux coming from an element can be approximated by its own emission and the reflections of solar and atmospheric radiations. This assumption is justified for the part of the second order reflected fluxes that is small compared to the total spectral reflected flux (Poglio et al., 2001). This approximation can not be done if material is very reflective, like aluminum. Without any specific user's requirements, the simulator operates in this manner. Second, third and subsequent reflection orders can be considered at user will. The output of the simulator (S2) is the 3-D scene for which the emittance of each element is known depending on the viewing direction.

The fourth primary simulator generates an image for a given viewing angle. Depending on the user preference, this primary simulator generates an image as it would be seen by the sensor or an image which is only a visualization of the 3-D scene. The first option requires another simulator to model the acquisition system. This can be done using the AS ${ }^{3}-\mathrm{I}$ simulator of Alcatel Space Industries. The second option offers a visualization of the 3-D scene. It permits to see the scene without any alteration due to the acquisition system.

The set of these four primary simulators constitutes a simulator of image in the infrared range. The user of such a simulator can be interested on not only the final image, but also on other additional outputs. The element-based representation of the scene and the wind flow over the 3-D scene (S0 outputs), the surface temperature of each element in the scene (S1 output) and the emitted flux or the reflected flux (S2 outputs) for example, are additional outputs. The simulator offers these options and several ancillary outputs are proposed, depending on user's requirements. These 
outputs can be used for the training of future users, and help the user to relate each of the numerous initial conditions to a change in the final image.

\section{CONCLUSION}

The physical process underlying the emitted flux in thermal infrared is very complex. The recent history of the landscape is present in the simulated image. The accuracy of the models of the physical processes and their interactions should be high in order to obtain a good quality. Consequently, a new methodology has been devised to design a simulator of landscape described by a 3-D representation. The concept of element was defined; it permits to describe with an accurate manner the objects of the landscape with respect to the physical processes and their variations in time and interactions. The architecture of the simulator was adapted to this concept of element and to the specificity of the simulation in the thermal infrared range. The simulator is made of four primary simulators, each of them having a welldefined role with respect to the description of the landscape on the physical processes. Preliminary experiments have shown the relevance of such a new methodology. Unavoidably it requests considerable efforts in landscape modeling, software development and computational resources, compared to simulation in the visible range.

\section{REFERENCES}

1. ASTER, 2000. ASTER spectral library Ver 1.2, CD-ROM, Jet Propulsion Laboratory, NASA, October 2000, http://spectib.jpl.nasa.gov/archive/jhu.html.

2. Barillot, P., June 2001, MISTRAL, http://www.onera.fr/dota/mistral/index.html.

3. Deardorff J.W., 1978. Efficient prediction of ground surface temperature and moisture with inclusion of a layer of vegetation. Journal of Geophysical Research, 83, C4, 1889-1902.

4. Foley J.D., van Dam A., Feiner S. K., Hughes J. F., 1996. Computer Graphics, Principles and Practice. Second Edition in C. Addison-Wesley Publishing Company, ISBN 0-201-84840-b, USA.

5. Guillevic P., 1999. Modélisation des bilans radiatif et énergétique des couverts végétaux. Thèse de Doctorat, Université P. Sabatier, Toulouse, France, 181 pp.

6. Jaloustre-Audouin K., 1998. SPIRou : Synthèse de Paysage en InfraRouge par modélisation physique des échanges à la surface. Thèse de Doctorat, Université de Nice-Sophia Antipolis, Nice, France, 169 pp.

7. Jaloustre-Audouin K., Savaria E., Wald L., 1997. Simulated images of outdoor scenes in infrared spectral band, AeroSense'97, SPIE, Orlando, USA.

8. Johnson K., Curran A., Less D., Levanen D., Marttila E., Gonda T., Jones J., 1998. MuSES: A new heat and signature management design tool for virtual prototyping, In Proceedings of the $9^{\text {th }}$ Annual Ground Target Modelling \& Validation Conference, Houghtoon, MI.

9. Johnson K.R., Wood S.B., Rynes P.L., Yee B.K., Burroughs F.C., Byrd T.,1995. A methodology for rapid calculation of computational thermal models, SAE International Congress \& Exposition, Underhood Thermal Management Session, Detroit, Michigan.

10. Kneizys F.X., Shettle E.P., Gallery W.O., Chetwind J.H., Abreu L.W., Selby J.E., Clough S.A., Fenn R.W., 1988. Atmospheric transmittance/radiance: computer code LOWTRAN 7 (AFGL-TR-88-0177), Hanscom AFB Massachusetts, Air Force Geophysics Laboratories, USA.

11. Louis J.-F., 1979. A parametric model of vertical eddy fluxes in the atmosphere. Boundary Layer Meteorology, 17 , 187-202.

12. Nicodemus F.E., Richmond J.C., Hsia J.J., 1977. Geometrical considerations and nomenclature for reflectance, NBS Monograph 160, U.S. National Bureau of Standards, Washington, D.C.

13. Noilhan J., Planton S., 1989. A simple parametrization of land surface processes for meteorological models. Monthly Weather Reviews, 117, 536-549.

14. Olseth A.J., Skartveit A., Czeplak G., Rommel M., 1994. Review and test of parametrizations of atmospheric radiation. A technical report of task 17. Measuring and modelling spectral radiation affecting solar systems and buildings. December 1994, International Energy Agency. Report n IEA SHCP 17F 2, 124 p.

15. Poglio T., Savaria E., Wald L., 2001. Influence of the three-dimensional effects on the simulation of landscapes in thermal infrared. To appear in proceedings of the $21^{\text {st }}$ Symposium EARSeL, Marne-la-Vallée, France.

16. Rigollier C., Bauer O., Wald L., 2000. On the clear sky model of the ESRA - European Solar Radiation Atlas - with respect to the Heliosat method. Solar Energy, 68 (1), 33-48. 
17. Schröder P., and Hanrahan P., 1993. On the form factor between two polygons. In Computer Graphics Proceedings, Annual Conference Series: SIGGRAPH'93 (Anaheim, CA), 163-164. ACM SIGGRAPH, New York.

18. Sillion F.X., and Puech C., 1994. Radiosity \& Global Illumination. Morgan Kaufmann Publishers, Inc., ISBN 1558-60277-1, San Francisco, CA, U.S.A., 251 p.

19. Thirion J.-P., 1991. Realistic 3-D simulation of shapes and shadows for image processing. CVGIP: Graphical Models and Image Processing, 54 (1), 82-90.

20. Watt A., 2000. 3-D Computer Graphics, Third Edition, Addison-Wesley Publishing Compagny Inc, ISBN 0-20139855-9. 